\title{
EDITORIAL
}

\section{CONTROLLED TRIALS OF PHYSIOTHERAPY}

It has been obvious for some time that the comfortable era of empirical physiotherapy has come to an end. On each physiotherapist rests the responsibility for ensuring that every treatment a patient receives is not only safe but also effective and efficient. This entails full examination of the patient, selecting the most suitable technique or modality and evaluating it in terms of effect or outcome.

For this to come about, controlled clinical trials, along similar lines to those employed in evaluating drug therapy, will have to be set up. Such trials will $\because$, valid only if the parameters by which efficacy is measured are reasonable in terms of expected outcome, the conditions being treated by physiotherapy have been shown to respond to it and a minimum of bias is introduced.

Some interesting and apparently contradictory examples of such trials have appeared in recent medical literature. Graham and Bradley (N. Engl. J. Med. 299, $624-627,1978$ ) assessed the efficacy of chest physiotherapy and intermittent positive pressure breathing (IPPB) in the resolution of pneumonia in a randomized clinical trial. They concluded that there was no statistical difference between the control group and treated groups, the latter having received IPPB concurrent with postural drainage, percussion and vibration. Parameters measured were duration of fever, extent of radiographic clearing, duration of hospital stay and mortality. This trial confirmed something which physiotherapists have believed, without controlled proof, for many years.

In another study, Newton and Stephenson (Lancet 2, $228-229,1978$ ) describe the effect of physiotherapy on pulmonary function. Elaborate laboratory tests were done on patients receiving physiotherapy whilst suffering from an acute exacerbation of chronic bronchitis. They concluded that there was no statistical difference in the pulmonary function of the two groups. Thus the controversy over the role of physiotherapy in chest disease remains, but further investigation is being undertaken.

A condensed report by Sims-Williams et al. (Brit. Med. J., 2, 1338-1340, 1978) of a controlled trial of mobilisation and manipulation for patients with low back pain in general practice once more seems to be inconclusive in that, although the treated group showed more immediate improvement, there was no significant difference between the two groups after a year.

These controlled studies should not discourage physiotherapists, but should serve as stimulation for further study. Expertise in research methodology and critical review of current knowledge and practice should enable the physiotherapist to establish which techniques can be applied to the benefit of the patient, to select those conditions which respond effectively to physiotherapy and to improve or modify existing techniques to achieve positive result.

If a scientific approach is added to the ability to establish warm human contact with patients, physiotherapy can only go from strength to strength.

\section{Contents - Inhoud}

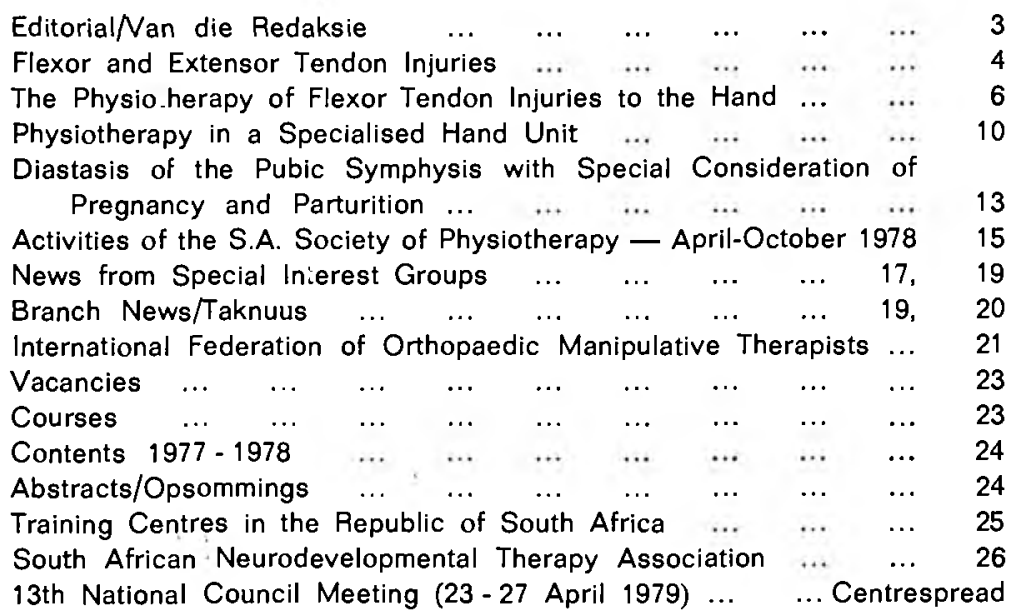

